\title{
DYNAMICAL FRICTION IN PAIRS OF ELLIPTICAL GALAXIES
}

\author{
Philippe Prugniel \\ European Southern Observatory \\ Karl-Schwarzschild-Str. 2 \\ D-8046 Garching, F.R.G. \\ Françoise Combes \\ Observatoire de Paris, DEMIRM \\ 2, Pl. J. Janssen \\ F-92195 Meudon, France
}

\begin{abstract}
We present numerical experiments on dynamical friction in pairs of elliptical galaxies of unequal mass. We confirm that the self-gravity of the response is not important and show the drastic effect of the deformability of the companion which reduces the decay time by more than a factor 2. Almost the same amount of orbital energy is dissipated within the satellite than within the large galaxy. Finally, we discuss the importance of distant encounters for the dynamical evolution of systems of galaxies.
\end{abstract}

\section{Introduction}

This communication presents the first results from a $\mathrm{N}$-body study of pairs of elliptical galaxies, they concern the investigation of the effect of various parameters on the dynamical friction in unequal mass pairs.

The important problem of the orbital decay of a "small" satellite onto a massive galaxy has been the object of a very open controversy over the last decade. The debate include the question of the globality of the response of the large galaxy and that of the importance of its self-gravity (Lin and Tremaine, 1983; White, 1983; Bontekoe and van Albada, 1987 (=BvA); Zaritsky and White, 1988 $(=\mathrm{ZW})$; Hernquist and Weinberg, 1989). Although the most recent publications agree on the merging time and, to a very satisfying accuracy, on the shape of the decay curve (separation versus time), the actual interpretation of the physical phenomena is still discussed. According to BvA, the dynamical friction can be characterized as a local process and the global response is not dynamically important. ZW agree about this last point, but claim for the globality of the nature of the dynamical friction although it can be described by a local formula. This discussion obviously suffers from the lack of clear and well accepted definitions of the terms. In this respect, the recent claim by Weinberg (1989) of the strong importance of the self-gravity, in the sense it increases the decay time by a factor 2 , is very confusing. Indeed, Weinberg includes in the self-gravity the motion of the center of gravity of the large galaxy while the other only consider the effect of the deformations on the internal potential.

In the present work we concentrate on the case of the friction at distance (when the two galaxies are well separated) which seems important for describing the earliest stages of the decay since the loose random encounters are strongly more usual in a cluster environment than the close ones. In addition, unlike previous work except Borne (1984), our companion is represented by a system of particles, and thus has the capability to absorb orbital energy as the large galaxy does.

The next part presents the experimental setup. The incidence of two physical ingredients is studied in 3 and 4: The effects of self-gravity and freedom degrees in the satellite. Then the importance of the friction at distance is investigated.

\section{Experimental setup}

We started from a self-gravitating system of two spherical galaxies since the $a$ priori allowed approximations were not obvious. Since the internal potential of the galaxies is computed on a 
cubic grid of finite resolution, we added a rigid core in order to simulate the high central density peak characteristic of elliptical galaxies without using a too large number of particles. The equations of motion are:

$$
\begin{gathered}
\ddot{x}_{c 1}=m_{1} \sum_{k 1}\left(x_{k 1}-x_{c 1}\right) p_{k c}^{-3}+m_{2} \sum_{l 2}\left(x_{l 2}-x_{c 1}\right) p_{l c}^{-3}+m_{c 2}\left(x_{c 2}-x_{c 1}\right) p_{c c}^{-3} \\
\ddot{x}_{j 1}=m_{1} \sum_{k 1}\left(x_{k 1}-x_{j 1}\right) p_{k j}^{-3}+m_{2} \sum_{l 2}\left(x_{l 2}-x_{j 1}\right) p_{l j}^{-3}+\cdots \\
+m_{c 1}\left(x_{c 1}-x_{j 1}\right) p_{c 1 j}^{-3}+m_{c 2}\left(x_{c 2}-x_{j 1}\right) p_{c 2 j}^{-3}
\end{gathered}
$$

Where we adopted the notations from White (1983), the indices 1 and 2 refers to each galaxy and should be inverted to obtain the accelerations of the second galaxy, $m_{1}$ and $m_{2}$ are the masses of the particles and $m_{c 1}$ and $m_{c 2}$ those of the cores.

For technical reasons the interaction between the particles' of the two galaxies (second term of $1 \mathrm{~b}$ ) is approximated by a multipolar expansion (White, 1983). This approximation is a crucial point, but since for the experiments presented here we have made further simplifications which avoid the difficulty, we will not address the point.

Two cases are considered: (1) a rigid satellite. (2) a satellite represented by $\mathrm{N}$-particles, without self-gravity.

In case (1) the second terms of Eq. $1 \mathrm{a}$ and $\mathrm{b}$ are ignored, and all the mass of the satellite is concentrated in the rigid potential. This makes the code formally equivalent to the classical problem of the decay of a satellite. The energy, angular and linear momentum are conserved.

In case (2) the two first terms of Eq. $1 \mathrm{~b}$ are replaced by the interaction with fixed potential:

$$
\sum_{k 1} m_{1} \nabla \Phi_{1}\left(x_{j 1}\right)+\sum_{l 2} m_{2} \nabla \Phi_{2}\left(x_{j 1}\right)
$$

The problem is then that considered by Borne (1984), and it does not formally conserve energy.

In our code, running on the CCVR CRAY 2, each galaxy is made by 20000 particles, and the selfgravitating potential is computed on a cubic $60 \times 60 \times 60$ points grid. Forces are derived and interpolated from this grid. In the case (2) only the symmetric spherical part of the initial (relaxed) potential is taken into account.

The initial model has a central rigid Plummer containing $1 / 3$ of the total mass and the particles are distributed according to a truncated $1 / \mathrm{r}^{2}$ density profile. The velocities follow an isotropic maxwellian distribution truncated at the escape velocity. Then, each galaxy is virialized separately, and the final density profile appears reasonably realistic. An important step, as stressed by Borne (1984), is the initial relaxation in the tidal field of the second galaxy before starting the interaction. We chose to not relax the initial galaxies in the actual initial tidal field, since such procedure is likely to exaggerate the phase coupling and the amplitude of the tidal bulges. Instead, we relax the galaxies in a spherically averaged tidal field. This has the advantage to avoid the energy shock responsible for strong transient effects (affecting the decay time by a factor two in Borne's experiments).

The mass ratio between the two galaxies lies in the range 3 to 10 , typical of the distorted pairs of ellipticals presented by Prugniel and Davoust (this meeting). The mass of the primary galaxy is $10^{12} M_{\odot}$ and its radius $30 \mathrm{kpc}$, the radius of the companion is $10 \mathrm{kpc}$.

In all the experiments presented here the companion was launched on a circular orbit of radius in the range $30-60 \mathrm{kpc}$. 
As a check of our procedure we run an experiment comparable to the standard experiment defined by White (1983). The decay curves are compared in Fig. 1. The agreement on the merging time is very good, but there are some difference in the shape of the decay curves. Although we did not check it, we believe the differences are due to the different mass profiles between these experiments.

Figure 1: Comparison with White (1983) experiment: decay of a pointmass satellite onto a large galaxy from a circular grazing orbit. The mass ratio is 10 and the mass of the large galaxy is $10^{12} M_{\odot}$. The broken line is White's data and the full line is our experiment.

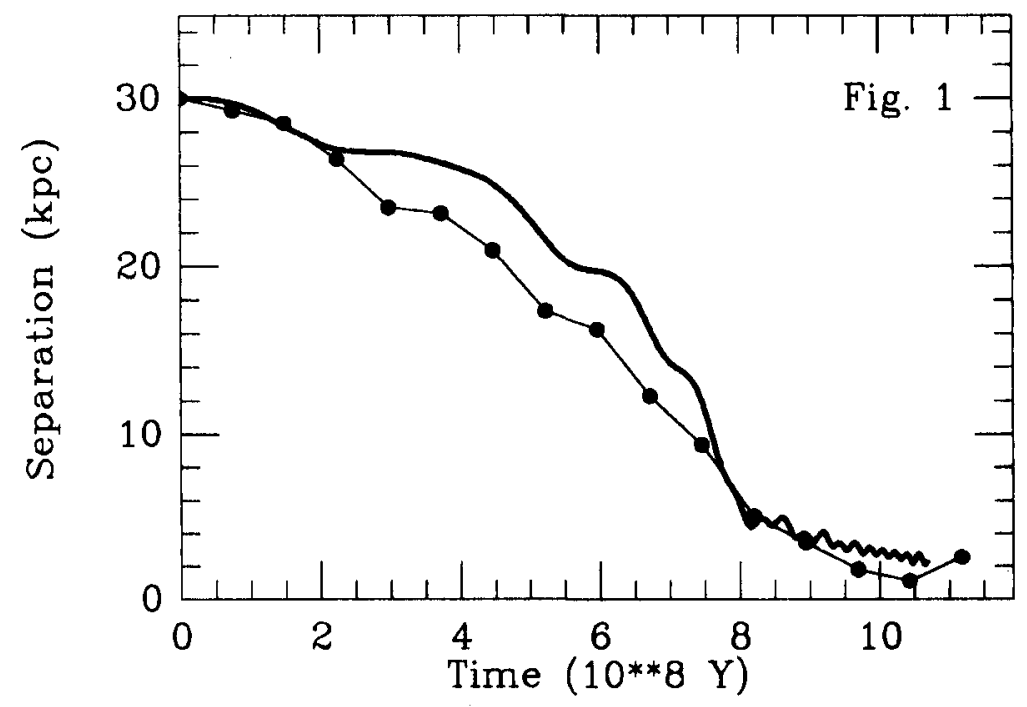

\section{Effect of the self-gravity of the response}

The first point investigated is the role of the self-gravity of the response. This is done by comparing experiments with the version (1) of the code with other done with version (2) but with a rigid satellite. Fig. 2 presents the decay curves for these experiments. The differences are not larger than the dispersion between experiments started from various initial conditions. This confirms the results of BvA and ZW and shows the consistency of the two versions of the code.

Figure 2: Effect of the self-gravity of the response of the large galaxy. Decay of a point mass satellite: same experimental setup as for Fig. 1. The full line includes the self-gravity of the deformation (case 1) while the dashed line does not (case 2). The difference is not significant.

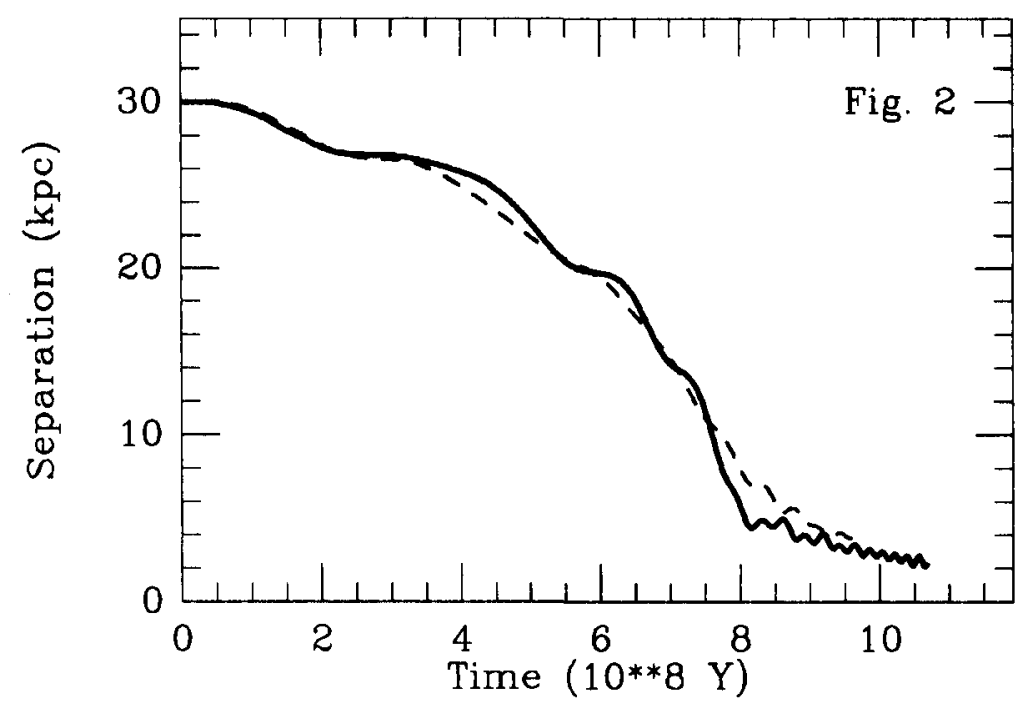




\section{Effect of the deformability of the companion}

The second point is the analysis of the effect of the ability of the satellite to absorb energy. Fig. 3 shows the results. The effect is considerable: the merging time is reduced by a factor 2.5 and scanning the range of realistic values for the size of the satellite it is always decreased by a factor 2 to 3 .

Figure 3: Effect of the deformability of the satellite. Decay of an extended satellite (radius $10 \mathrm{kpc}$ ) starting on a circular orbit. The masses are $10^{12} M_{\odot}$ and $10^{11} M_{\odot}$, the radius of the large galaxy is $30 \mathrm{kpc}$. The full line is for a rigid satellite and in the experiment represented by a dashed line both galaxies are made with 20000 particles.

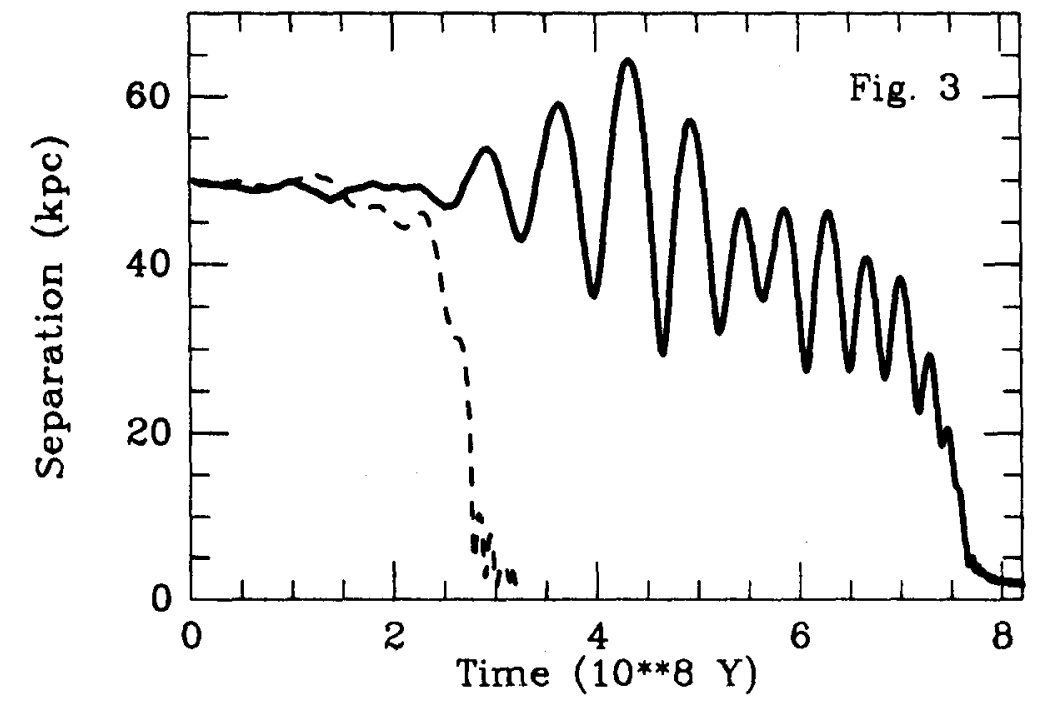

\section{Importance of the friction at distance}

Clearly, the classical formula for the dynamical friction does not apply when the galaxies are well separated (since in this model the drag is proportional to the local density). However, it is important to know the relative importance of loose encounter over close encounter for what concern the evolution of groups of galaxies. The alternative approach is the impulsive approximation (Spitzer, 1958), but it is not suitable to our problem where the relative velocity is of the same order or even smaller than the typical internal velocities. However, Dekel et al. (1980) have shown that the impulsive approximation still gives results compatible with numerical experiments for slow hyperbolic encounters.

We have conducted experiments starting from distant circular orbits. The difficulty of these experiments with low friction is the high sensitivity to the noise due to the limited number of particles. With 20000 particles, we were able to run experiments up to an initial orbital radius of $60 \mathrm{kpc}$ (with a rigid satellite). The initial decay rates $(d R / d t)$ are compared with the prediction of the impulsive approximation on Fig. 4. The dashed line corresponds to the classical impulsive approximation (Spitzer, 1958; $d R / d t \propto R^{-3}$ ) while the full line is the restriction to the drag due to the particles with a velocity smaller than the encounter velocity $\left(d R / d t \propto R^{-9 / 2}\right)$. 
Figure 4: Dependence of the initial decay rate $(d R / d t)$ on the radius of the orbit. The masses are $10^{12} M_{\odot}$ and $10^{11} M_{\odot}$ and the satellite is rigid. The dashed line corresponds to the classical formula for impulsive approximation (Spitzer, 1958), in principle not suitable to this case, and the full line is the friction computed under the impulsive approximation restricted to the low velocity particles.

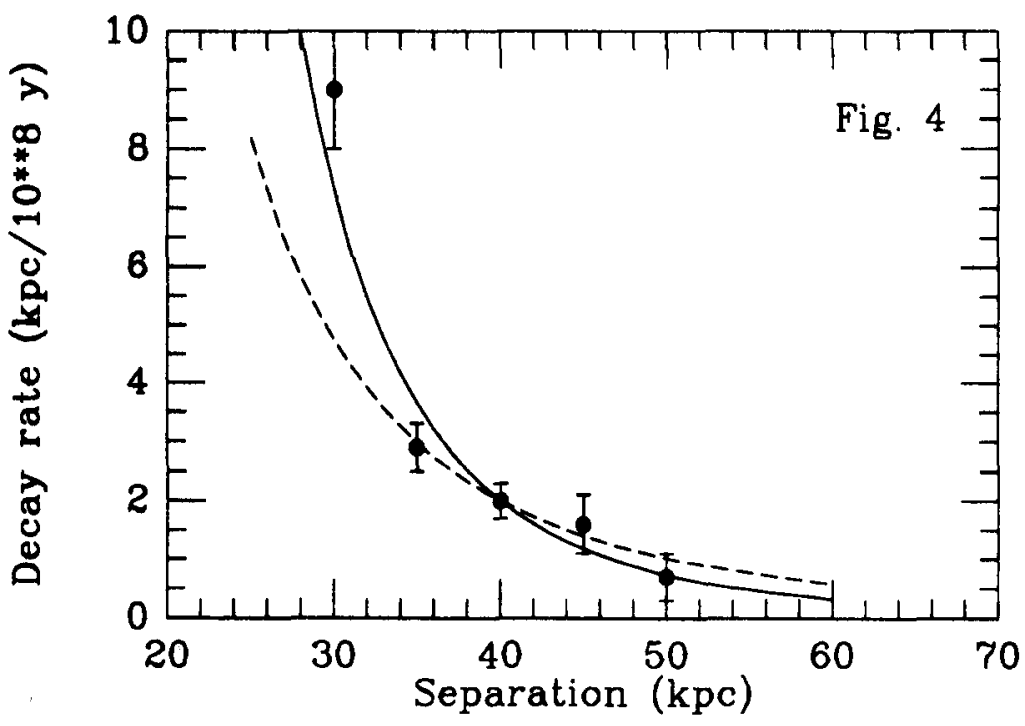

\section{Conclusions}

We have confirmed that the self-gravity of the response is not important and revealed the drastic effect of the ability of the small companion to absorb orbital energy. The amount of energy absorbed by the satellite is comparable to that dissipated within the large galaxy. This effect is associated to the very strong deformation of the satellite illustrated in Fig. 5. The distortion pattern of the simulated pairs looks very much like the one observed in real pairs (e.g. Davoust and Prugniel, 1988 or Prugniel and Davoust, this meeting). As for the global-local character of the friction phenomenon, we investigate this question in a subsequent publication (Prugniel and Combes, 1990).

Figure 5: Tidal distortion of the galaxies. This plot shows the density of particles in a logarithmic scale at an advanced stage of one of our experiments. The initial separation was $40 \mathrm{kpc}$ and the mass ratio is 10 . The field represented is $120 \mathrm{kpc}$ wide and the actual separation is $10 \mathrm{kpc}$. The merging will occur in $5 \times 10^{7}$ years. Note the extended diffuse tail behind the satellite and the characteristic pattern of distortion symmetric with respect to the pair center.

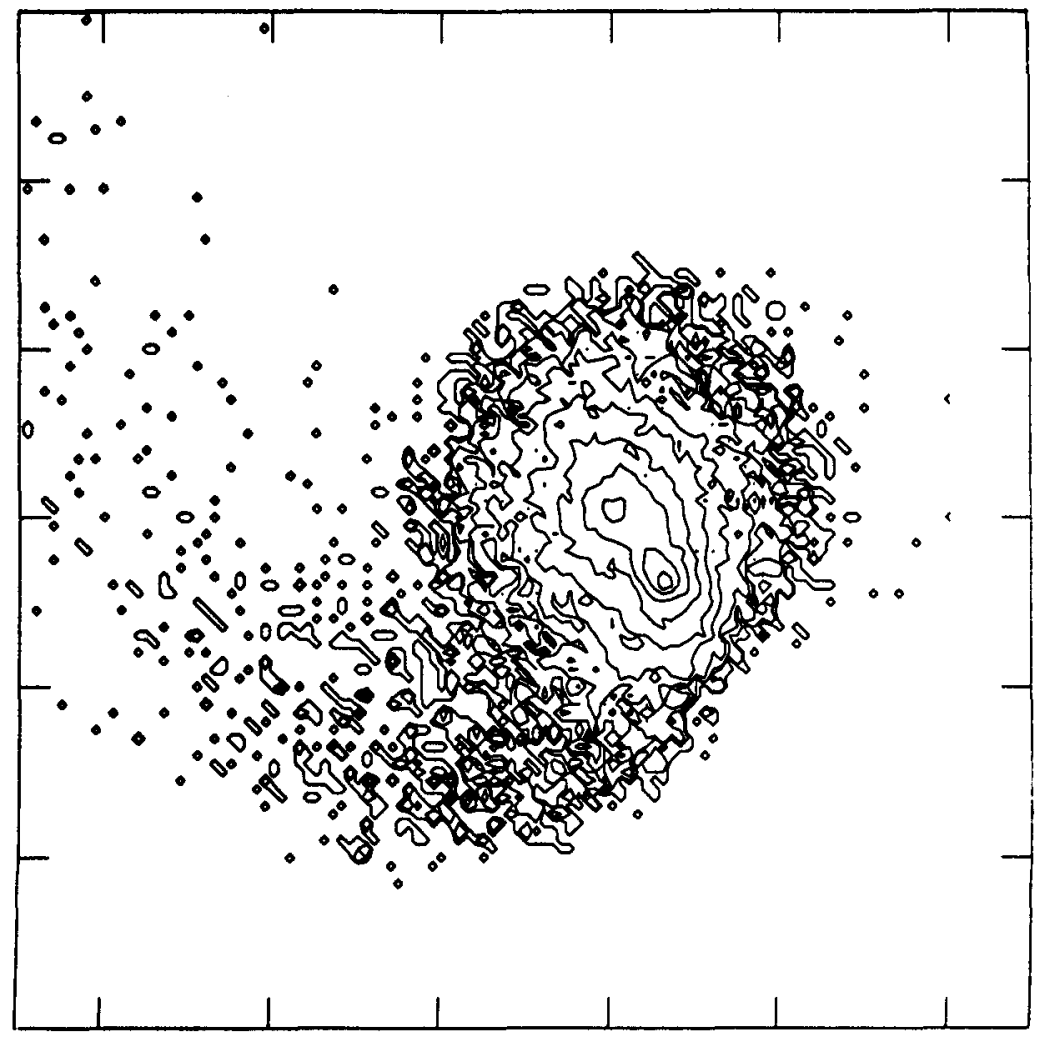




\section{References}

Bontekoe, Tj.R., van Albada, T.S.: 1987 (BvA), Mon. Not. Roy. Ast. Soc. 224, 349

Borne, K.D.: 1984, Astrophys. J. 287, 503

Davoust, E., Prugniel, Ph.: 1988, Astron. Astrophys. Letters 201, L30

Dekel, A., Lecar, M., Saham, J.: 1980, Astrophys. J. 241, 946

Hernquist, L., Weinberg, M.D.: 1989, Mon. Not. Roy. Ast. Soc. 238, 407

Lin, D.N.C., Tremaine, S.: 1983, Astrophys. J. 264, 364

Spitzer L.: 1958, Astrophys. J. 127, 17

Weinberg, M.D.: 1989, Mon. Not. Roy. Ast. Soc. 239, 549

White, S.D.M.: 1983, Astrophys. J. 274, 53

Zaritsky, D., White, S.D.M.: 1987 (ZW), Mon. Not. Roy. Ast. Soc. 235, 289 